if the number of patients is small. Further studies are needed to identify a reliable predictive marker of uveitis risk in JIA patients. The finding of a significant greater prevalence of anti-DFS70 autoantibodies in healthy ANA + subjects allows to suppose that this autoantibody could represent a possible protective marker for development of AARDs in asymptomatic children with isolated ANA positivity, as for adults. To confirm this hypothesis, it would be useful to carry on the study prospectively, encompassing children with other rheumatological diseases, and prolonging the clinical and laboratory follow up.

References:

[1] Clarke S, Sen ES, Ramanan AV. Juvenile idiopathic arthritis-associated uveitis. Ped Reumatol 2016; 14: 27.

[2] Seeling CA, Bauer O, Seeling H-P. Autoantibodies Against DFS70/LEDGF Exclusion Markers for Systemic Autoimmune Rheumatic Diseases (SARD). Clin. Lab 2016;62:499- 517.

[3] Schmeling $\mathrm{H}$ et al. Autoantibodies to Dense Fine Speckles in Pediatric Diseases and Controls. The Journal of Rheumatology 2015;42(12):2419-2426.

Disclosure of Interests: None declared

DOI: 10.1136/annrheumdis-2020-eular.1815

\section{AB1004 JUVENILE DERMATOMYOSITIS (JDM) IN SOUTHEAST ASIA: A 20-YEAR SINGAPORE EXPERIENCE}

K. L. Teh ${ }^{1}$, L. Das ${ }^{1}$, Y. X. Book ${ }^{1}$, T. Arkachaisri ${ }^{1,2} .{ }^{1} K K$ Women's and Children's Hospital, Rheumatology and Immunology, Singapore, Singapore, ' ${ }^{2}$ Duke-NUS Medical School, Singapore, Singapore

Background: Juvenile dermatomyositis (JDM) is a multisystem inflammatory disease of childhood with variable demographics, clinical features and outcomes. No studies have described the characteristics of JDM patients from Southeast Asia population.

Objectives: To describe the clinical characteristics and outcomes of JDM patients in Singapore over a 20 -year period.

Methods: Patients diagnosed with JDM from 1999 to 2019 at KK Women's and Children's Hospital, Singapore, were recruited. Nonparametric descriptive statistics were used to described data. Kaplan-Meier analyses were used to estimate the probability of remission. Multivariate logistic and Cox regression analyses were used to determine predictors as appropriate. The significant level was set at $<0.05$

Results: 32 JDM were identified. Clinical characteristics and treatment used are shown in Table 1.

\begin{tabular}{lccc}
\hline & All $(\mathrm{n}=32)$ & Monophasic $(\mathrm{n}=17)$ & Polyphasic $(\mathrm{n}=14)$ \\
\hline Male & $14(43.8)$ & $7(41.2)$ & $7(50.0)$ \\
Age at onset (yrs)* & $6.4(4.5-9.8)$ & $5.4(4.1-8.5)$ & $7.4(5.6-12.3)$ \\
Lag period (mo) & $3.5(1.0-12.5)$ & $2.0(1.0-16.8)$ & $6.8(1.2-15.8)$ \\
Heliotrope & $16(50)$ & $9(52.9)$ & $7(50)$ \\
Gottron papule & $23(71.9)$ & $12(70.6)$ & $11(78.6)$ \\
Malar rash & $19(61.3)$ & $9(52.9)$ & $10(71.4)$ \\
Vasculitic rash & $19(61.3)$ & $8(47.1)$ & $11(78.6)$ \\
Arthritis & $10(31.3)$ & $3(17.6)$ & $7(50)$ \\
Nailfold changes & $28(87.5)$ & $15(88.2)$ & $13(92.9)$ \\
Calcinosis & $9(28.1)$ & $4(23.5)$ & $5(35.7)$ \\
Positive ANA & $17(53.1)$ & $10(58.8)$ & $7(50)$ \\
Positive Myositis antibodies & $4(12.5)$ & $2(11.8)$ & $2(14.3)$ \\
Laboratory at diagnosis, U/L ${ }^{*}$ & & & \\
CK & $324(134-2229)$ & $746(139-2965)$ & $351(155-2622)$ \\
LDH & $650(450-943)$ & $650(398-1015)$ & $714(512-944)$ \\
ALT & $28(16-106)$ & $35(18-109)$ & $38(15-104)$ \\
AST & $50(30-108)$ & $50(31-113)$ & $85(36-215)$ \\
Aldolase & $13.7(8.1-28.0)$ & $14.2(8.4-26.6)$ & $16.3(8.6-38.5)$ \\
CMAS score at diagnosis & $29(21-43)$ & $28(21-35)$ & $35(11-43)$ \\
\hline
\end{tabular}

${ }^{*}$ median (IQR), otherwise - n (\%)

Pulse methylprednisolone (pMP) was used in $53.1 \%$ of patients after diagnosis. Median time to inactive disease (ID) was 5.3 months (IQR $2.8-12.8$ ). Male, older age and patients on pMP ( $p=0.003-0.044)$ achieved ID sooner. Older patients also developed disease flare sooner after achieving ID ( $p$ $=0.024)$. No clinical features nor lab investigations predicted JDM disease course. Malay patients was associated with higher risk of calcinosis $(p=$ 0.017).

Compared to adult dermatomyositis patients in Singapore ${ }^{1}$, our cohort had more cutaneous manifestations including malar rash, vasculitic rash and nailfold changes. Table 2 shows the time for each muscle enzymes to normalise.

\begin{tabular}{lc}
\hline CK & $1.49(0.69-3.53)$ \\
LDH & $4.53(2.35-25.04)$ \\
ALT & $1.71(0.90-4.13)$ \\
AST & $0.97(0.53-3.12)$ \\
Aldolase & $3.12(2.35-8.30)$
\end{tabular}

median in months (IQR)

Conclusion: Our cohort of JDM patients had more calcinosis compared to othe Asian population ${ }^{2}$. Malay population is at higher risk of this complication. It is crucial to achieve ID state in the shortest time possible to avoid significant morbidity. Our study suggests that early treatment with pMP is associated with shorter time to ID. There is no predictor identified for disease course, similar to previous studies $^{3}$.

\section{References:}

[1] Liu, Wen Chun, et al. "An 11-year review of dermatomyositis in Asian patients." Annals Academy of Medicine Singapore 39.11 (2010): 843.

[2] Sun, Chi, et al. "Juvenile dermatomyositis: a 20-year retrospective analysis of treatment and clinical outcomes." Pediatrics \& Neonatology 56.1 (2015): 31-39.

[3] Stringer, Elizabeth, Davinder Singh Grewal, and Brian M. Feldman. "Predicting the course of juvenile dermatomyositis: significance of early clinical and laboratory features." Arthritis \& Rheumatism: Official Journal of the American College of Rheumatology 58.11 (2008): 3585-3592.

Disclosure of Interests: None declared

DOI: 10.1136/annrheumdis-2020-eular.5929

\section{AB1005 1 BULLOUS LUPUS (BSLE) AS THE FIRST MANIFESTATION OF SYSTEMIC LUPUS ERYTHEMATOSUS IN THE PEDIATRIC POPULATION (PSLE): A DIAGNOSTIC CHALLENGE IN DAILY PRACTICE}

F. Torres ${ }^{1}$, M. Velasquez ${ }^{1,2}$, L. Ramirez ${ }^{3}$, L. Leon ${ }^{4}$, M. Martinez ${ }^{1} .{ }^{1}$ Universidad de Antioquia, Medellin, Colombia; ${ }^{2}$ Hospital San Vicente Fundacion, Medellin Colombia; ${ }^{3}$ Hospital Fundacion Valle del Lili, Cali, Colombia; ${ }^{4}$ Universidad Nacional de Colombia, Bogota, Colombia

Background: Cutaneous manifestations are observed in $59-85 \%$ of patients with SLE but less than 5\% developed BSLE. In the GLADEL cohort, the prevalence is $0.41 \%$. BSLE literature in children is scarce
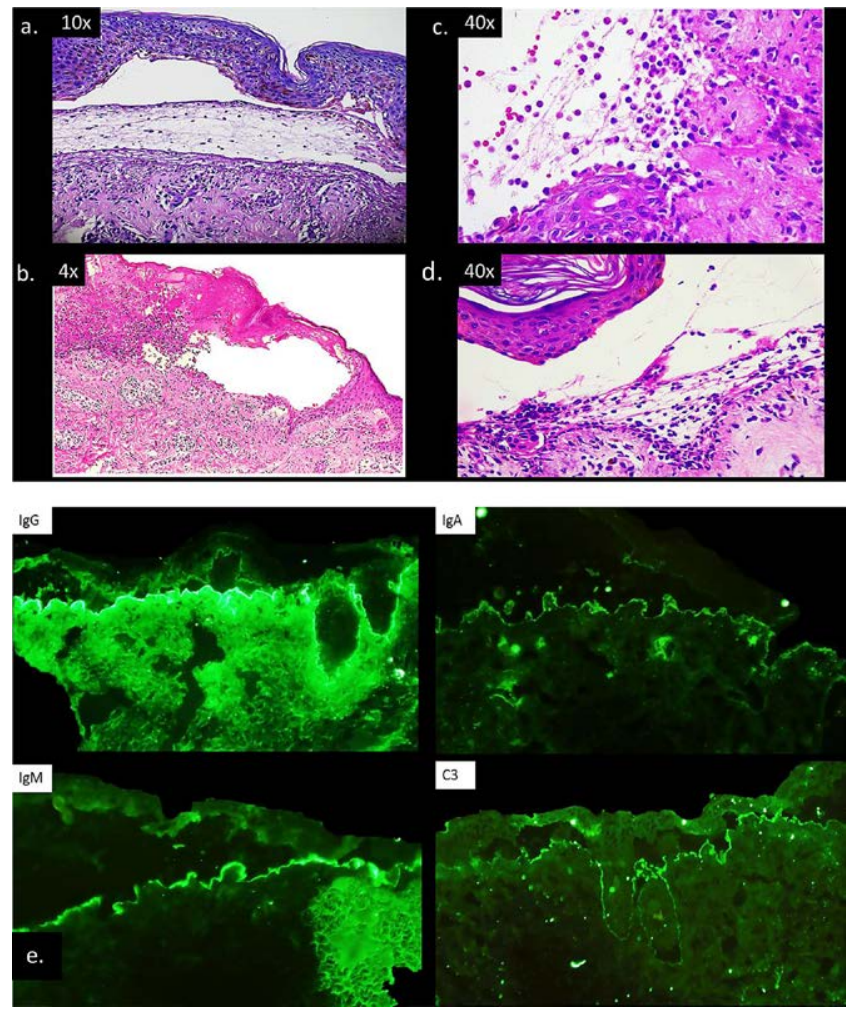

Fig 1. Histology and DIF of BSLE H-E $\mathbf{a}$ and $\mathbf{b}$ : subepidermal blisters $\mathbf{c}$ and $\mathbf{d}$ : abundant neutrophils are observed e: DIF full house linear pattern 